\title{
COUPLED CLUSTERS AND THE ONE-COMPONENT BOSE PLASMA
}

\author{
R.F. Bishop and K.H. Lührmann
}

Department of Mathematics

University of Manchester Institute of Science and Technology

P.0. Box 88, Manchester M60 1QD, England

\begin{abstract}
We show that the coupled cluster formalism in its lowest level of approximation provides an excellent description of the one-component Bose plasma at all densities. In particular, using a translationally-invariant description, we derive a unified framework that both exactly describes the high-density plasma and at least qualitatively describes the low-density solid phase.
\end{abstract}

A general discussion of the coupled cluster formalism of many-fermion theory and its application to the electron gas has recently been given by the present authors [1]. Attention was focussed there on the high-density limit, and in order to make further progress we have now also studied the analytically much simpler, although conceptually very similar, Bose formalism.

In essence, the coupled-cluster formalism employs the linked-cluster theorem for the exact many-body ground-state wavefunction $|\psi\rangle$ (i.e., the usual exp(S) Ansatz) to decompose the Nbody system into a set of mutually interacting $n$-body subsystems $(n \leq N)$ that are described by a set of so-called correlation operators $S$, the matrix elements of which describe the excitation of $n$ "particle-hole pairs" from some suitably defined non-interacting reference (or model) state $\mid \Phi>$. Although it is not necessary to be so restrictive we choose $|\Phi\rangle$ here to be a single-state condensate, and we have

$$
\begin{aligned}
&|\Psi\rangle= e^{S}|\Phi\rangle, S=\sum_{n=2}^{N} S_{n}, \\
&|\Phi\rangle=(N !)^{-\frac{1}{2}}\left(b_{0}^{\dagger}\right)^{N} \mid O>, \\
& S_{n}=\frac{1}{n !} \sum_{\rho_{1}} \ldots \rho_{n} b_{1}^{+} \ldots b_{\rho_{n}}^{+} S_{n}\left(\rho_{1} \ldots \rho_{n}\right)\left(N^{-\frac{1}{2}} b_{0}\right)^{n},
\end{aligned}
$$

where the operators $b_{\alpha}^{\dagger}$ create bosons in a complete orthonormal single-particle (s.p.) set $|\alpha\rangle$ in general, and which henceforth we choose as plane waves. The exact $\mathrm{N}$-body Schrödinger equation for the ground state is thereby exactly decomposed into a coupled set of $\mathrm{N}$ (non-linear) microscopic equations for the matrix elements of the correlation operators $s$. In the present case where we are interested ${ }^{\mathrm{n}}$ only in microscopic two-body (Coulomb) forces, and choosing $|\Phi\rangle$ to be the usual (translationally-invariant) zeromomentum condensate; the exact two-body equation (for spin-zero bosons) for $\mathrm{S}_{2}(q) \equiv \mathrm{S}_{2}(\vec{q},-\vec{q})$ becomes,

$\frac{\hbar^{2} \mathrm{q}^{2}}{\mathrm{~m}} \mathrm{~S}_{2}(\mathrm{q})+\mathrm{T}_{\mathrm{RPA}}+\mathrm{T}_{\mathrm{CP}}+\mathrm{T}_{\mathrm{LAD}}+\sum_{\mathrm{q}^{\prime}} \mathrm{V}\left(\mathrm{q}^{\prime}\right)$

$x\left[2 N^{\frac{1}{2}} S_{3}\left(\vec{q}, \vec{q}^{\prime},-\vec{q}-\vec{q}^{\prime}\right)+\frac{1}{2} N S_{4}\left(\vec{q},-\vec{q}, \vec{q}^{\prime},-\vec{q}^{\prime}\right)\right]=0$,

$$
\begin{aligned}
& \mathrm{T}_{\mathrm{RPA}}=\mathrm{NV}(\mathrm{q})\left[1+\mathrm{S}_{2}(\mathrm{q})\right]^{2}, \mathrm{~T}_{\mathrm{CP}}=-4 \frac{E_{\mathrm{N}}}{\mathrm{S}_{2}(\mathrm{q}),} \\
& \mathrm{T}_{\mathrm{LAD}}=\sum_{\overrightarrow{\mathrm{q}}^{\prime}} \mathrm{V}\left(\overrightarrow{\mathrm{q}}-\overrightarrow{\mathrm{q}}^{\prime}\right) \mathrm{S}_{2}\left(\mathrm{q}^{\prime}\right),
\end{aligned}
$$

and where the g.s. energy is given by

$$
E / N=\frac{1}{2} N V(0)+\frac{1}{2} \sum_{q} V V(q) S_{2}(q) \text {. }
$$

The density $\rho \equiv N / \Omega$ is expressed in terms of either the usual dimensionless coupling constant $r$, which is the average interparticle spacing in units of the Bohr radius $a$, or a fictitious Fermi wavenumber $k$ applicable to an analogous

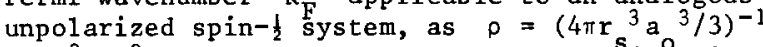
$=\mathrm{k}_{\mathrm{F}}{ }^{3} / 3 \pi^{2}$; and the g.s. energy per particle is expressed in Rydberg units as $E / N=\varepsilon\left(e^{2} / 2 a_{0}\right)$. The so-called SUB2 approximation is obtained from Eq. (2) by setting $\mathrm{S}_{3}$ and $\mathrm{S}_{4}$ to zero. The remaining four terms then represent respectively (i) the kinetic energy (KE) contribution; (ii) the terms that, with $\mathrm{KE}$, generate the randomphase approximation (RPA); (iii) the terms that generate the fully self-consistent (s.c.) energy insertions on the zero-momentum condensate lines, viz., the s.c. condensate potential (CP); and (iv) the terms that repeatedly scatter two particles outside the condensate and hence generate the two-particle ladder (LAD) diagrams. Where all dimensionless momenta are scaled against the fictitious Fermi momentum $\mathrm{hk}_{\mathrm{F}}$, the SUB2 equation for Coulomb forces becomes,

$$
\begin{gathered}
q^{2} S_{2}(q)+4 \alpha r_{s}\left(3 \pi q^{2}\right)^{-1}\left[1+S_{2}(q)\right]^{2}-2\left(\alpha r_{s}\right)^{2} \varepsilon S_{2}(q) \\
+\frac{\alpha r}{\pi q} \int_{0}^{\infty} d q^{\prime} q^{\prime} \ln \left|\frac{q^{\prime}+q}{q^{\prime}-q}\right| S_{2}\left(q^{\prime}\right)=0, \\
\varepsilon=2\left(\pi \alpha r_{s}\right)^{-1} \int_{0}^{\infty} d q S_{2}(q) ; \alpha^{3}=4 / 9 \pi .
\end{gathered}
$$

Although the nonlinear equation (4) is readily solved numerically, it is more instructive to examine its high- and low-density limits. In the high-density $\left(r_{s} \rightarrow 0\right)$ limit it is trivial to show that $\varepsilon \rightarrow Q r-3 / 4+R ;$ and furthermore that on $1 y$ the $\mathrm{KE}$ and $\mathrm{RPA}$ terms contribute to leading order to give the well-known exact result $Q \approx-0.8031$ of Foldy [2]. The $\mathrm{CP}$ and $\mathrm{LAD}$ terms also contribute to next order to give a SUB2 value $R=16 / 9 \pi$. Inspection of Eq. (2) shows that the coupling 
terms to $\mathrm{S}_{3}$ and $\mathrm{S}_{4}$ also contribute to the constant $\mathrm{R}^{3}$ (a1though not to Q). A careful and detailed calculation including these terms leads to the value $R \approx 0.0280$, and an intricate rearrangement shows that our result is identical to the first correct result reported [3], of Brueckner.

In the much more interesting strong-coupling limit, $r \rightarrow \infty$, one may not expect the SUB2 approximation to give a reasonable result, since one imagines that $n$-body clusters with $n>2$ are of importance here. Indeed we expect the system to undergo a phase transition to a Wigner solid, and this crystal phase is an archetype of one where $\mathrm{N}$-body correlations dominate. However, in the SUB2 approximation we find that $\varepsilon \rightarrow$ $-\mathrm{Ar}^{-1}+\mathrm{Br}^{-3 / 2}+\mathrm{O}\left(\mathrm{r}^{-2}\right)$ as $\mathrm{r} \rightarrow \infty$, and that the $\mathrm{KE}$ term contributes only to the constant $B$ in leading order. Whereas each of the terms RPA, CP and LAD is necessary for a quantitative evaluation of the constant $A$, they play quite different physical roles. Whereas the RPA terms continue to be vital for the correct analytic behaviour by providing the correet long-range $(q \rightarrow 0)$ Coulomb screening, the $C P$ now plays a similarly crucial role in the short-range $(q \rightarrow \infty)$ limit. Whereas the inclusion of the LAD term quantitatively changes the constants $A$ and $B$, it may safely be omitted without changing this analytic form for the low-density energy expansion. Dropping the LAD term leads to the analytic results $A=\left(32 / 3 \pi^{2}\right)^{1 / 3} \approx 1.03 ; B=3^{\frac{1}{2}} \pi / 8 \approx$ 0.68 . The virial theorem may also be used to show that in this low-density expansion the leading term is purely potential energy, whereas the much more interesting second term is half each kinetic and potential energy - a result strongly indicative of the simple harmonic motion (SHM) expected of a solid. Indeed, Wigner first pointed out that in this limit, where the potential energy dominates, the energy is minimized by the particles crystallizing; and this leads to an electrostatic energy proportional to $x^{-1}$. Whereas in a fluid phase the particles are free to occupy the whole volume and hence by the uncertainty principle have a kinetic energy proportional to $\mathrm{r}^{-2}$; the particles in the Wigner solid are constrained to oscillate about fixed lattice sites and hence to have a greater kinetic energy, which by simple SHM considerations is seen to vary as $r^{-3} / 2$. Thus our SUB2 g.s. energy has the correct analytic form of a solid, even though the coefficients are not very close to the b.c.c. crystal values of $A \approx 1.792$, $B \approx 2.65$. (We note that inclusion of the LAD term only increases this discrepancy by reducing the value of $A$ by about $20 \%$.) It is important to realize however that even the lowest SUB2 approximation in the coupled cluster scheme gives a low-density energy which cannot possibly represent a fluid phase since the particles are not free to occupy the whole volume.

It is clear therefore that our everywhere translationally-invariant approach well describes both fluid and solid phases. Although one might be surprised that the fluid-like SUB2 wavefunction is capable of describing an (amorphous) solid state, it is clear that strong many-body correlations are built into the approximate wavefunction on the other hand it is clear that in the lowdensity limit the third- and higher-order correlations obtained from the couplings to $S$ with $\mathrm{n}>2$ are still very strong, and ought to be incorporated for a better quantitative description. Due to the relative simplicity of the Bose coupled-cluster equations, this remains quite feasible to do (as indeed we have already shown in the high-density limit), and we hope to report later on such further results.

\section{REFERENCES}

[1] Bishop, R.F. and Lïhrmann, K.H., Phys.Rev. B17 (1978) 3757.

[2] Foldy, L.L., Phys.Rev. 124 (196I) 649; 125 (1962) 2208.

[3] Brueckner, K.A., Phys.Rev. 156 (1967) 204. 